\title{
Aspartic acid aminotransferase activity is increased in actively spiking compared with non-spiking human epileptic cortex
}

\author{
STEPHEN J KISH, LORI M DIXON, ALLAN L SHERWIN \\ From the Clarke Institute of Psychiatry, Toronto, and the Montreal Neurological Hospital and Institute, \\ Montreal, Canada
}

SUMMARY Increased concentration of the excitatory neurotransmitter aspartic acid in actively spiking human epileptic cerebral cortex was recently described. ${ }^{2}{ }^{3}$ In order to further characterise changes in the aspartergic system in epileptic brain, the behaviour of aspartic acid aminotransferase (AAT), a key enzyme involved in aspartic acid metabolism has now been examined. Electrocorticography performed during surgery was employed to identify cortical epileptic spike foci in 16 patients undergoing temporal lobectomy for intractable seizures. Patients with spontaneously spiking lateral temporal cortex $(n=8)$ were compared with a non-spiking control group $(n=8)$ of patients in whom the epileptic lesions were confined to the hippocampus sparing the temporal convexity. Mean activity of AAT in spiking cortex was significantly elevated by $16-18 \%$, with aspartic acid concentration increased by $28 \%$. Possible explanations for the enhanced AAT activity include increased proliferation of cortical AAT-containing astrocytes at the spiking focus and/or a ? generalised increase in neuronal or extraneuronal metabolism consequent to the ongoing epileptic discharge. It is suggested that the data provide additional support for a disturbance of central excitatory aspartic acid mechanisms in human epileptic brain.

We have recently reported an increase in the concentration of two excitatory amino acid neurotransmitters, aspartic acid and glutamic acid, in actively spiking lateral temporal cortex excised for neurosurgical therapy of focal epilepsy. ${ }^{23}$ These changes may be especially relevant with respect to the pathophysiological processes in human focal cortical epilepsy in view of the experimental evidence that activation of central aspartic acid/glutamic acid systems may induce, in some brain areas, both seizure activity and concomitant neuronal damage. ${ }^{45}$ Taken together, these findings support the notion that sei-

Address for reprint requests: Stephen J Kish, Human Brain Laboratory, Clarke Institute of Psychiatry, 250 College Street, Toronto, Ontario M5T 1R8, Canada.

Received 4 August 1987 and in revised form 21 October 1987. Accepted 26 October 1987 zures and seizure-mediated brain damage are associated with excessive activation of excitatory amino acid receptors.

In order to clarify further the status of the aspartic acid neuronal system we have now examined in biopsied human epileptic brain the behaviour of aspartic acid aminotransferase (AAT, EC 2.6.1.1), a key enzyme involved in both aspartic acid as well as glutamic acid metabolism. ${ }^{6}$ This enzyme catalyses the reversible conversion of oxaloacetic acid and glutamic acid to aspartic acid and alpha-ketoglutarate. We found a significant increase in AAT activity together with above-normal levels of aspartic and glutamic acid in spiking vs. non-spiking epileptic temporal cortex.

\section{Patients and methods}

Patients

Electrocorticography with surface and depth electrodes was 
employed during surgery to identify areas of focal epileptic activity in 16 patients undergoing temporal lobectomy for the therapy of intractable seizures. Antiepileptic drugs were partially or completely withdrawn to enhance the recording of epileptic activity at the time of operation which was performed under local anaesthesia. Spontaneously active widespread epileptic activity was present in eight patients with lateral temporal cortical foci (S1 to S8, see table). In eight other cases (N9 to N16), the epileptic activity recorded (approximately 30 minutes duration) did not involve the surface of the temporal lobe, but was restricted to the hippocampus. By anatomical and therapeutic necessity these superficial nonspiking regions were also included in the standard "en bloc" temporal lobectomy for intractable seizures. Tissue was excised from either the middle or superior temporal gyrus (Brodman areas 21, 22, and 38) within anterior 5-6 $\mathrm{cm}$ of temporal cortex. The surgical specimens were immediately placed on ice and from one to six samples (approximately $200 \mathrm{mg}$ ) carefully dissected from blood vessels and extraneous tissue, wrapped in aluminium foil, frozen on dry ice within 15 minutes of excision, and immediately stored at $-80^{\circ} \mathrm{C}$. After excision of tissues for biochemical analysis adjacent regions of cortex and white matter were fixed and stained by the Kluver-Barrera and Cajal Gold chloride sublimate techniques for neurons and glia respectively. As shown in the table the mean age of the patients with actively spiking cortex was not significantly different from that for the non-spiking patients ( $p>0.05$, Student's two-tailed test). The type (see table) and duration of the anticonvulsant medication were similar for both patient groups.

All biochemical determinations were conducted blind.

\section{Enzyme and amino acid assays}

For the assay of total (cytosolic and mitochondrial) AAT, biopsied brain was homogenised in 600 volumes of $0.1 \mathrm{M}$ potassium phosphate buffer $(\mathrm{pH} 7.5)$ containing $0.5 \%$ Triton X-100. All incubation mixtures contained $600 \mu \mathrm{l}$ homogenate, $100 \mu$ l of potassium phosphate buffer $(0.41 \mathrm{M}$, $\mathrm{Ph} 7.5$ ) and $10 \mu \mathrm{l}$ of $12.5 \mathrm{mM}$ pyridoxal-5-phosphate. For assays in the direction of aspartic acid formation incubation mixtures also contained $190 \mu 1$ of $0.313 \mathrm{M}$ potassium glutamate, which included $0.2 \mu \mathrm{Ci}$ of $\mathrm{U}-{ }^{14} \mathrm{C}$-glutamate (New England Nuclear) and either $100 \mu 1$ of $30 \mathrm{mM}$ oxaloacetic acid (Sigma) or (for blanks) water. In the direction of aspartic acid degradation incubation mixtures contained $190 \mu \mathrm{l}$ of $0.215 \mathrm{M}$ potassium aspartate which included $0.2 \mu \mathrm{Ci} \mathrm{U}-{ }^{14} \mathrm{C}$ aspartate (New England Nuclear) and either $100 \mu \mathrm{l}$ of $0.2 \mathrm{M}$ potassium alpha-ketoglutarate or (for blanks) $100 \mu \mathrm{l}$ of water. After a 10 minute incubation at $37^{\circ} \mathrm{C}$ the reaction was stopped by adding $100 \mu \mathrm{l}$ of $2 \mathrm{~N} \mathrm{HCl}$. The ${ }^{14} \mathrm{C}$-labelled alpha-ketoglutarate formed (aspartic acid formation) or ${ }^{14} \mathrm{C}$-labelled oxaloacetate formed (aspartic acid

Table Patient information, aspartic acid aminotransferase and amino acid values: spiking vs. non-spiking temporal cortex

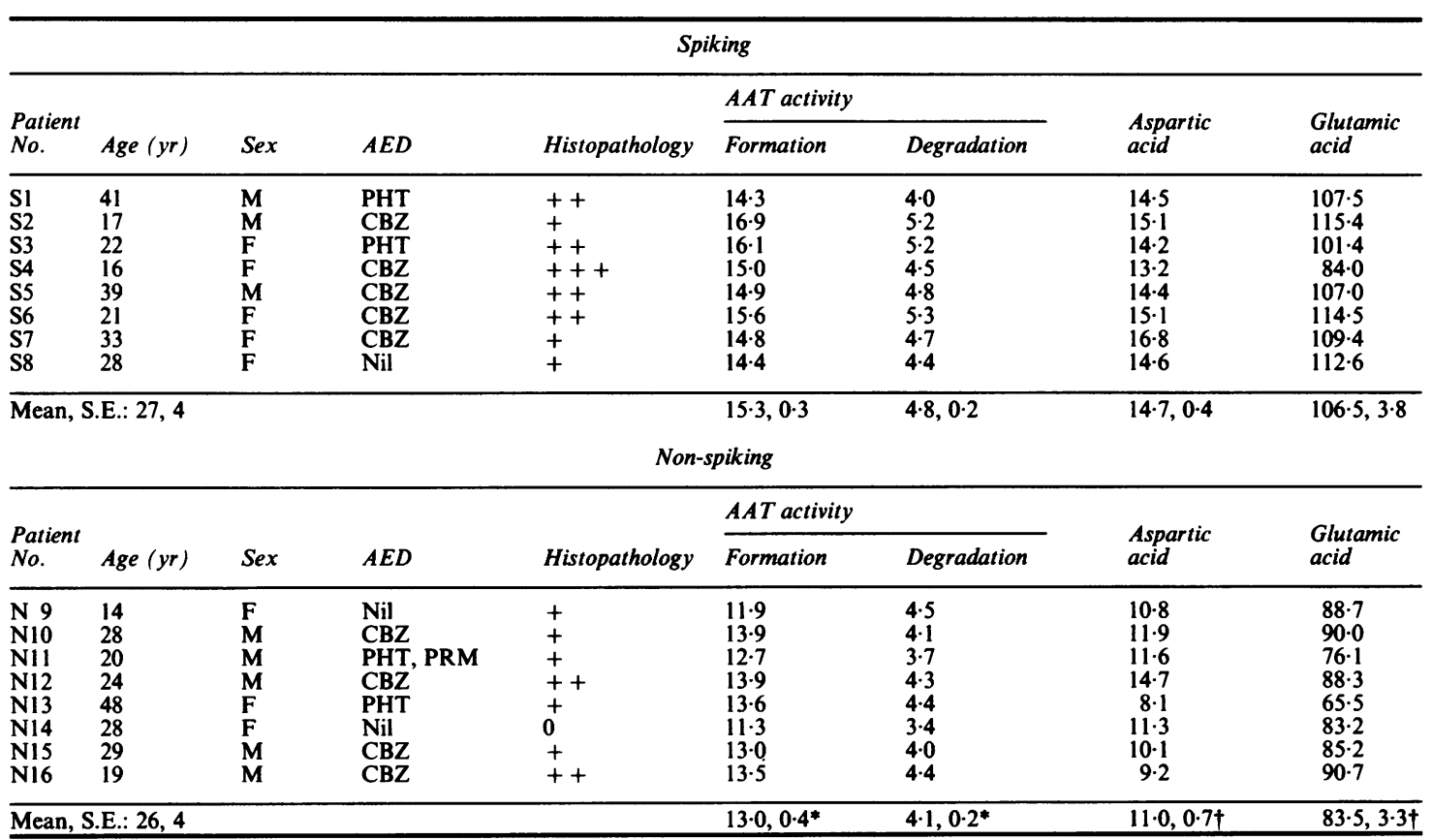

*p $<0.01$. tp $<0.001$; Student's two-tailed $t$ test.

Abbreviations: AAT, aspartic acid aminotransferase; AED, antiepileptic medication; CBZ, carbamazepine; PB, phenobarbital; PHT, phenytoin; PRM, primidone; Nil, anti-epileptic drug completely eliminated prior to surgery. 0 , no evidence of astroglial proliferation, small benign lesion in hippocampus. + , very mild astrogliosis without evidence of neuronal loss; ++ , mild to moderate astrogliosis and slight neuronal loss;,+++ marked proliferation of fibrous astrocytes with neuronal loss involving all cortical lamina. AAT activity was measured in the direction of aspartic acid formation and degradation. Values are expressed as mmol/g protein/10 min (AAT) and $\mathrm{nmol} / \mathrm{mg}$ protein (aspartic and glutamic acid). 
degradation) were separated from their radioactive precursors by applying incubation mixtures to $5 \times 0.75 \mathrm{~cm}$ columns of Dowex $50-\mathrm{X} 8$ resin, $\mathrm{H}^{+}$form (Sigma) and eluted with $3.0 \mathrm{ml}$ of water. The eluates were then counted for radioactivity. The enzyme assays were linear with respect to time and protein homogenate concentration over the range studied. Amino acids were quantitated using minor modifications of the procedure of Fernstrom and Fernstrom. $^{7}$

\section{Results}

As shown in the table, AAT activity measured in the direction of aspartic acid formation and degradation was significantly increased by 18 and $16 \%$ respectively, in spiking vs non-spiking cortex. In spiking cortex concentrations of aspartic and glutamic acid were significantly elevated by $34 \%$ and $28 \%$ respectively. An analysis of the individual values for AAT revealed little overlap between the spiking and non-spiking groups (especially in the direction of aspartic acid formation) with the majority of the spiking values falling outside the range of the non-spiking levels (see fig). When the biochemical data from all 16 patients were subjected to regression analysis a significant positive correlation was observed between AAT activity measured in the direction of aspartic acid formation and in the direction of degradation $(\mathrm{r}=0.83 ; \mathrm{p}<0.001)$. AAT activity was also significantly correlated with concentrations of aspartic acid (formation, $\mathrm{r}=0.65 ; \mathrm{p}<0.005$; degradation, $r=0.48 ; p<0.05$ ) and glutamic acid (formation, $r$ $=0.66 ; \mathrm{p}<0.005$; degradation, $\mathrm{r}=0.61 ; \mathrm{p}<0.01$ ).

Specimens from patients with superficial cortical spike foci tended to exhibit a greater degree of cortical astrogliosis and neuronal loss than those from the

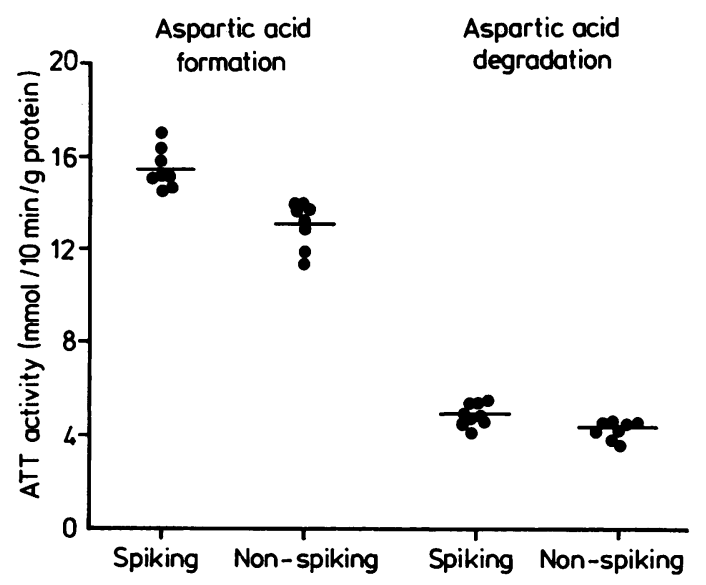

Fig Activity of aspartic acid aminostransferase in the direction of aspartic acid formation and degradation in actively spiking vs. non-spiking human epileptic cortex. patients with deep temporal lesions. Thus five patients in the spiking group showed grade ++ or +++ histopathology. vs. only two patients in the non-spiking group.

\section{Discussion}

To our knowledge, this study describes, for the first time, the behaviour of AAT in actively spiking human epileptic brain.

Our observation of above normal AAT activity in human epileptic cortex may be related to a generalised increase in neuronal and/or extraneuronal metabolic activity present in the epileptic focus. Ir this regard, in biochemical studies of human epileptic cortex elevated activities of other enzymes involved in neurotransmitter metabolism, namely, glutamate dehydrogenase ${ }^{8}$ tyrosine hydroxylase ${ }^{8}$ cholineacetyltransferase (Kish and Sherwin, unpublished observations), and acetylcholinesterase (refs. 9, 10; Kish and Sherwin, unpublished observations) have been demonstrated. In this context, the possibility also has to be considered that the above-normal AAT levels in spiking cortex may be causally related to enhanced astroglial proliferation and activity. In this respect we observed in the 16 patients examined a slight, but statistically significant correlation between the activity of AAT in the direction of aspartic acid formation and extent of the histopathological changes $\left(r_{s}=\right.$ $0.52, p<0.02$, Spearman Rank Order Correlation; $p$ $>0.05$ for histopathology vs. AAT in direction of aspartic acid degradation, aspartic acid or glutamic acid concentration). The enzyme AAT has been demonstrated to be present in especially high activities in astrocytes (11, cf. ref. 12).

In our study all antiepileptic medication had been partially or completely withdrawn prior to operation. Most of the patients had received carbamazepine monotherapy and in all cases plasma levels had been in the lower therapeutic range for several weeks to permit EEG localisation. However, the possibility that these drugs even in low dosage may have influenced the amino acid and enzyme values cannot be excluded. None had received valproate which the acute administration of high doses in experimental animal studies is reported to result in a reduction in brain aspartate levels. ${ }^{1314}$ Aspartic acid concentration in cerebrospinal fluid has been reported to be higher in epileptic patients receiving antiepileptic polytherapy vs. patients without any medication. ${ }^{15}$

With respect to functional considerations, much evidence now suggests the involvement of the excitatory neurotransmitter aspartic acid in seizure conditions as well as seizure-related neuronal damage.

Sloviter and Dempster ${ }^{4}$ have provided evidence 
that in rodents repeated intraventricular injection of aspartic acid produces both seizures and morphological changes in the hippocampus which were qualitatively similar to the changes observed in temporal lobe of epileptic patients. Similarly, Piredda and Gale $^{5}$ have recently demonstrated the ability of aspartic acid to induce bilateral motor seizures when microinjected into the deep prepiriform cortex of the rat. In the experimental literature studies of the behaviour of brain aspartic acid in a variety of animal models of convulsive seizures have produced no consistent pattern with aspartic acid levels reported as being reduced: cobalt focus, ${ }^{16-19}$ methoxypyridine, ${ }^{20}$ allylglycine, bicuculline, kainic acid, ${ }^{21}$ benzodiazepine inverse agonists, ${ }^{22}$ increased: pyridoxal phosphate, ${ }^{2324}$ sound-induced seizures, ${ }^{25}$ homocysteine $^{26}$ or normal: kindling, ${ }^{27-29}$ pentylenetetrazol. $^{30}$ However, investigations of the influence of experimental epileptic conditions on aspartic acid release have consistently shown enhanced release of this excitatory neurotransmitter. ${ }^{19222429}$ Additional evidence is derived from pharmacological studies demonstrating anticonvulsant properties of amino acid analogues which preferentially block the electrophysiological responses of aspartic acid and N-methyl-Daspartate $^{31-33}$ In this regard it may be especially noteworthy that such excitatory amino acid antagonists have recently been shown to block electrically stimulated bursting of action potentials in biopsied human epileptic cortex. ${ }^{34}$ Chapman and coworkers ${ }^{14}$ reported a strong correlation between the anticonvulsant potency of valproic acid analogues with their ability to reduce brain aspartic acid levels. These experimental data taken together with our present biochemical findings suggest the possibility of enhanced aspartic acid synthesis and neuronal release in actively spiking (but not with convulsions) epileptic human brain. This receives some additional support from the observations of increased aspartic acid levels in cerebrospinal fluid of epileptic patients. ${ }^{1535-37}$

Although the magnitude of the AAT elevation was slight $(+16-18 \%)$ we observed little overlap in enzyme values (especially in the direction of aspartic acid formation) between the two patient groups (see fig). It will therefore be interesting to determine whether in future studies employing this new research paradigm AAT activity can be shown to distinguish reliably between actively spiking vs. non-spiking epileptic temporal cortex.

Finally, we suggest that our biochemical observations implicating the brain aspartic acid system in cortical epilepsy further strengthen the rationale for the clinical testing of centrally acting excitatory amino acid antagonists in human epileptic conditions. $^{38}$
S K is a Career Scientist of the Ontario Ministry of Health.

Presented in part at the IBRO Second World Congress of Neuroscience, Budapest, August 1987 (ref. 1).

\section{References}

1 Dixon LM, Sherwin A, Olivier A, et al. Aspartate aminotransferase activity in actively spiking vs. non spiking human epileptic cerebral cortex (Abstract). Neuroscience 1987; 22(Suppl):568.

2 Sherwin A, Olivier A, Quesney F, Robitaille Y, Andermann E, Andermann F, Feindel W. Glutamate and aspartate but not GABA or taurine concentrations are elevated in actively spiking versus nonspiking human epileptic cerebral cortex (Abstract). Thirty-ninth Meeting of the American Academy of Neurology, April 5-11, 1987, New York. Neurology 1987;37(Suppl. 1):247.

3 Sherwin A, Robitaille Y, Quesney F, et al. Excitatory amino acids are elevated in human epileptic cerebral cortex. Neurology (in press).

4 Sloviter RS, Dempster DW. "Epileptic" brain damage is replicated qualitatively in the rat hippocampus by central injection of glutamate or aspartate but not by GABA or acetylcholine. Brain Res Bull 1985;15:39-60.

5 Piredda S, Gale K. Role of excitatory amino acid transmission in the genesis of seizures elicited from the deep prepiriform cortex. Brain Res 1986;377:205-10.

6 Cooper AJL. Glutamate-Aspartate Transaminase. Methods Enzymol 1985;113:66-9.

7 Fernstrom MH, Fernstrom JD. Rapid measurement of free amino acids in serum and CSF using high performance liquid chromatography. Life Sci 1981;29:2119-40.

8 Sherwin A, Quesney F, Gauthier S, et al. Enzyme changes in actively spiking areas of human epileptic cerebral cortex. Neurology 1984;34:927-33.

9 Pope A, Morris AA, Jasper H, Elliott KAC, Penfield W. Histochemical and action potential studies on epileptogenic areas of cerebral cortex in man and the monkey. Res Publ Ass Nerv Ment Dis 1947;26:218.

10 Tower DB, Elliott KAC. Activity of acetylcholine system in human epileptogenic focus. J Appl Physiol 1952;4:669.

11 Schousboe A, Svenneby G, Hertz L. Uptake and metabolism of glutamate in astrocytes cultured from dissociated mouse brain hemispheres. J Neurochem 1977;29:999-1005.

12 Yu AC, Schousboe A, Hertz L. Metabolic fate of 14C-labeled glutamate in astrocytes in primary cultures. $J$ Neurochem 1982;39:954-60.

13 Schechter PJ, Tranier Y, Grove J. Effect of n-dipropylacetate on amino acid concentrations in mouse brain: correlations with anti-convulsant activity. Neurochem 1978;31:1325-7.

14 Chapman AG, Meldrum BS, Mendes E. Acute anticonvulsant activity of structural analogues of valproic acid and changes in brain GABA and aspartate content. Life Sci 1983;32:2023-7.

15 Engelsen B, Elsayed S. Increased concentrations of aspartic acid in the cerebrospinal fluid of patients with epilepsy and trigeminal neuralgia: an effect of medication? Acta Neurol Scand 1984;70:10-76.

16 Van Gelder NM, Courtois A. Close correlation between changing content of specific amino acids in epileptogenic cortex of cats, and severity of epilepsy. Brain Res 1972;43:477-84.

17 Koyama U. Amino acids in the cobalt-induced epileptogenic and nonepileptogenic cat's cortex. Can J Physiol Pharmacol 1972;50:740-52.

18 Emson PC, Joseph MH. Neurochemical and morphological changes during the development of cobalt-induced epilepsy in the rat. Brain Res 1975;93:91-110. 
19 Abdul-Ghani AS. Effect of gamma-amino-4-phosphonobutyrate on the release of endogenous glutamate and aspartate from cortical synaptosomes of epileptic rats. $J$ Neurochem 1985;45:365-9.

20 Nitsch C, Schmude B, Haug P. Alterations in the content of amino acid neurotransmitters before the onset and during the course of methoxy-pyridoxine-induced seizures in individual rabbit brain regions. $J$ Neurochem 1983;40:1571-80.

21 Chapman AG, Westerberg E, Premachandra M, Meldrum BS. Changes in regional neurotransmitter amino acid levels in rat brain during seizures induced by L-allylglycine, bicuculline, and kainic acid. $J$ Neurochem 1984;43:62-70.

22 Chapman AG, Cheetham SC, Hart GP, Meldrum BS, Westerberg E. Effects of two convulsant beta-carboline derivatives, DMCM and B-CCM, on regional neurotransmitter amino acid levels and on vitro D- $\left[{ }^{3} \mathrm{H}\right]$ aspartate release in rodents. $J$ Neurochem 1985:45:370-81.

23 Wiechert P, Gollnitz G. Metabolic investigations of epileptic seizures. The concentration of free amino acids in cerebral tissue prior to and during cerebral seizures. $J$ Neurochem 1969;16:1007-16.

24 Norris DK, Murphy RA, Chung SH. Alteration of amino acid metabolism in epileptogenic mice by elevation of brain pyridoxal phosphate. $J$ Neurochem 1985;44:1403-10.

25 Chapman AG, Faingold CL, Hart GP, Bowker HM, Meldrum BS. Brain regional amino acid levels in seizure susceptible rats: changes related to sound-induced seizures. Neurochem Int 1986;8:273-9.

26 Allen IC, Grieve A, Griffiths R. Differential changes in the content of amino acid neurotransmitters in discrete regions of the rat brain prior to the onset and during the course of homocysteine-induced seizures. $J$ Neurochem 1986;46: 1582-92.

27 Fabisiak JP, Schwark WS. Cerebral free amino acids in the amygdaloid kindling model of epilepsy. Neuropharmacology
1982;21:179-82.

28 Leach MJ, Miller AA, O'Donnell RA, Webster RA. Reduced cortical glutamine concentrations in electrically kindled rats. $J$ Neurochem 1983;41:1491-4.

29 Leach MJ, Marden CM, Milller AA, O'Donnell RA, Weston SB. Changes in cortical amino acids during electrical kindling in rats. Neuropharmacology 1985;24:937-40.

30 Fabisiak JP, Schwark WS. Aspects of the pentylenetetrazol kindling model of epileptogenesis in the rat. Exp Neurol 1982;78:7-14

31 Meldrum BS, Croucher MJ, Badman G, Collins JF. Antiepileptic action of excitatory amino acid antagonists in the photosensitive baboon, papio papio. Neurosci Lett 1983;39:101-4.

32 Jones AW, Croucher MJ, Meldrum BS, Watkins JC. Suppression of audiogenic seizures in DBA/2 mice by two new dipeptide NMDA receptor antagonists. Neurosci Lett 1984;45:157-61.

33 Herron CE, Williamson $\mathrm{R}$, Collingridge GL. A selective $\mathbf{N}$ methyl-D-aspartate antagonist depresses epileptiform activity in rat hippocampal slices. Neurosci Lett 1985;61:255-60.

34 Avoli M, Olivier A. Bursting in human epileptogenic neocortex is depressed by an N-methyl-D-aspartate antagonist. Neurosci Lett 1987;76:249-54.

35 Plum CM. Free amino acid levels in the cerebrospinal fluid of normal humans and their variation in cases of epilepsy and spielmeyer-vogt-batten disease. J Neurochem 1974;23:595-600.

36 Weitz R, Merlob P, Amir J, Reisner SH. A possible role for aspartic acid in neonatal seizures. Arch Neurol 1981;38: 258-9.

37 Smith CCT, Bowen DM, Francis PT, Snowden JS, Neary D. Putative amino acid transmitters in lumbar cerebrospinal fluid in patients with histologically verified Alzheimer's dementia. J Neurol Neurosurg Psychiatry 1985;48:460-71.

38 Meldrum B. Excitatory amino acid antagonists as novel anticonvulsants. In: Excitatory Amino Acids and Epilepsy. Adv Exp Med Biol 1986;203:321-9. 